\title{
reviews
}

\section{Islands in space}

R. L. F. Boyd

The High Frontier: Human Colonies in Space. By G. K. O'Neill. Pp. 288. (Jonathan Cape: London, 1977.) £5.95.

A population of several thousand million people located in space colonies, within a mere 35 years of the initiation of a space colonisation programme, is typical of the thinkingone might even say the evangel-of Dr O'Neill. In this readable and extraordinarily optimistic promotion of the cause of space as better than this Earth the author argues that man must be persuaded to rid himself of his "planetary chauvinism" and accept that even the Moon or Mars. for example, would not provide as suitable an environment as man-made habitats situated at one of the semi-stable Lagrangian points in the Earth-Moon system.

Three stages of development are suggested. "Island One" would be constructed primarily of aluminium and glass inside a sphere of $0.5 \mathrm{~km}$ diameter. The habitat, filled with an oxygen atmosphere, would rotate to provide an Earthlike gravity at the equator. The great majority of materials for the construction would be mined and transported from the lunar surface. Heavy industry, located nearby outside the sphere, and agriculture would, it is claimed, benefit from the continuous and predictable supply of solar energy, and the whole system could support a population of 10,000 in some comfort. Careful screening would prevent any unwanted "bugs" from being taken in from Earth; and presumably no malign mutation is expected to occur.

Within a further 50 years, the scale of development from these small beginnings is envisaged as leading to the "Island Three" habitats, cylinders 4 miles in diameter and 20 miles in length, maintaining several million inhabitants. There is probably no reason to dispute that such a design is already within the limits of present-day structural materials. Economic motivation for such colonies is claimed to lie with the construction of large solar power stations, subsequently placed into geosynchronous orbit and providing the Earth with electricity through high power microwave transmission. Such systems have, of course,

already been the subject of detailed design studies under NASA sponsorship, but it is not clear why sunlight in space should be more economic than in the desert areas of the near tropic latitudes here.

This book may properly rank as space fiction rather than prophecy but if so it is not because of any violation of scientific laws. No fundamentally new physics is required nor any great projection of present-day engineering practice. The speed with which such plans could be formulated and implemented even if adequate political backing was forthcoming, as suggested by O'Neill, is a point on which opinion will, however, vary greatly. In particular, there is little discussion of the inevitable sociological, legal and personal, including moral and religious, questions in their widest sense.

Much space is devoted to the projected economic aspects of space colonisation, particularly in terms of the initial investment, and to considering the viability of selling power to Earth through the solar power stations, considerations inevitably of a very speculative nature and, in themselves, hardly a complete justification for this mammoth scheme.

In chapter 2, entitled "The Human Prospect on Planet Earth", O'Neill presents what he sees as the most compelling arguments for a massive

\section{Animal cells in culture}

Biochemical Methods in Cell Culture and Virology. By Robert J. Kuchler. Pp. ix +331 . (Halsted/Wiley: New York and Chichester, UK, 1977.) $£ 22.50 ; \$ 38$.

BECAUSE research in animal virology is based to a large extent on the use of animal cells in culture, it might seem a good idea to include in one manual the procedures of both. In fact, the task is not an easy one, as this review will suggest.

The stated aim of this book is to provide a guide for growing, handling and studying viruses and cell cultures in the hope that the student considering a career in virology, cell biology or cancer research should find it useful. There are three parts, of about equal lengths: Cell Culture, Virology, and colonisation of space. The shortage of land and energy on Earth are even now an obvious cause for concern and, with the expected rate of population growth, the situation may become desperate even before the middle of the next century. It may be thought, however, that these are basically political and ethical questions, that given good will, unselfishness, honesty, education, tolerance, sympathy and so on, it would be no more difficult to build a heaven on Earth than a "New Earth" (the title of chapter 4) in the heavens.

The New English Bible translates an aspiration of the early Christian church from two millenia ago, and no doubt echoing a far older yearning of many people, as "we . . . look forward to new heavens and a new Earth, the home of justice". This problem with its associated need for wisdom and moral fibre outweights all the technical difficulties we face. Solve this and we might avoid the energy crisis of the late 1980s and the serious risk of war between "haves" and "have-nots" before the end of then century.

R. L. F. Boyd is Professor of Physics in the University of London, and Director of the Mullard Space Science Laboratory, Department of Physics and Astronomy. University College, London, UK.
Macromolecular Analysis. The content is essentially a collection of very many procedures. Each procedure, or group of related procedures, is preceded by a brief introduction on its purpose and, very cursorily, on its history.

Not many students can secure a copy of the standard procedures, formulations, recipes, and so on, which several large research laboratories, dealing with virology and cell cultures, necessarily keep. These procedures are of ten collected in loose-leaf Laboratory Manuals. Every time that a procedure or formulation is changed, it is an easy matter to replace the corresponding pages.

The next best method (though expensive) for a student who cannot get hold of any such manual, is a book; but a book does not have replaceable 